\title{
Climate Refugia: Joint Inference from Fossils, Genetics and Models
}

\section{Eugene, USA, 1-3 August 2012}

\section{Daniel G. Gavin' ${ }^{1}$, S. Z. Dobrowski ${ }^{2}$, A. Hampe ${ }^{3}$, F. S. Hu4 and F. Rodriguez-Sanchez ${ }^{5}$}

'Department of Geography, University of Oregon, Eugene, USA; dgavin@uoregon.edu

2Department of Forest Management, University of Montana, Missoula, USA; ${ }^{3}$ Biodiversité, Gènes et Communautés, INRA, Bordeaux, France; ${ }^{4}$ Department of Plant Biology, University of Illinois, Urbana, USA; ${ }^{5}$ Department of Plant Sciences, University of Cambridge, UK

limate refugia, i.e. areas where popu- lations of a species avert extinction during periods of unfavorable regional climate, may play a central role in maintaining regional-to-global biodiversity. Paleoecologists and biogeographers have a long-standing interest in refugia as a means to explain latitudinal migration or stasis of the geographic distribution of a species through glacial periods and the resulting relictual distributions and endemism (Hampe and Jump 2011). More recently, the burgeoning field of phylogeography has demonstrated the value of genetic information to complement the fossil record in understanding past species distributions. This has lead to increased recognition that far more species than previously thought may have maintained small and scattered populations through the Last Glacial Maximum in refugia situated at relatively high latitude (Stewart et al. 2010). Indeed, a generally low rate of extinction attributable to the dramatic climate change of the Quaternary is not congruent with high rates of future extinction that are projected by many species distribution models (Botkin et al. 2007). The existence of climate refugia in heterogeneous landscapes may provide one solution to this conundrum and contribute to important assessments regarding the risks of future climate change to biodiversity. Specifically, a better knowledge of the functioning and dynamics of historical climate refugia can enhance our understanding of how current populations may react to future climate changes, help with identifying potential refugia for species of concern, and improve our understanding of biotic responses to continued climate warming (Keppel et al. 2012).

We convened a three-day workshop, with support from PAGES and the University of Oregon, to bring together experts from diverse disciplines that provide complementary insights into the identification, characterization, and importance of climate refugia. The workshop engaged 33 participants from 10 countries with backgrounds in paleoecology, climatology, phylogeography,

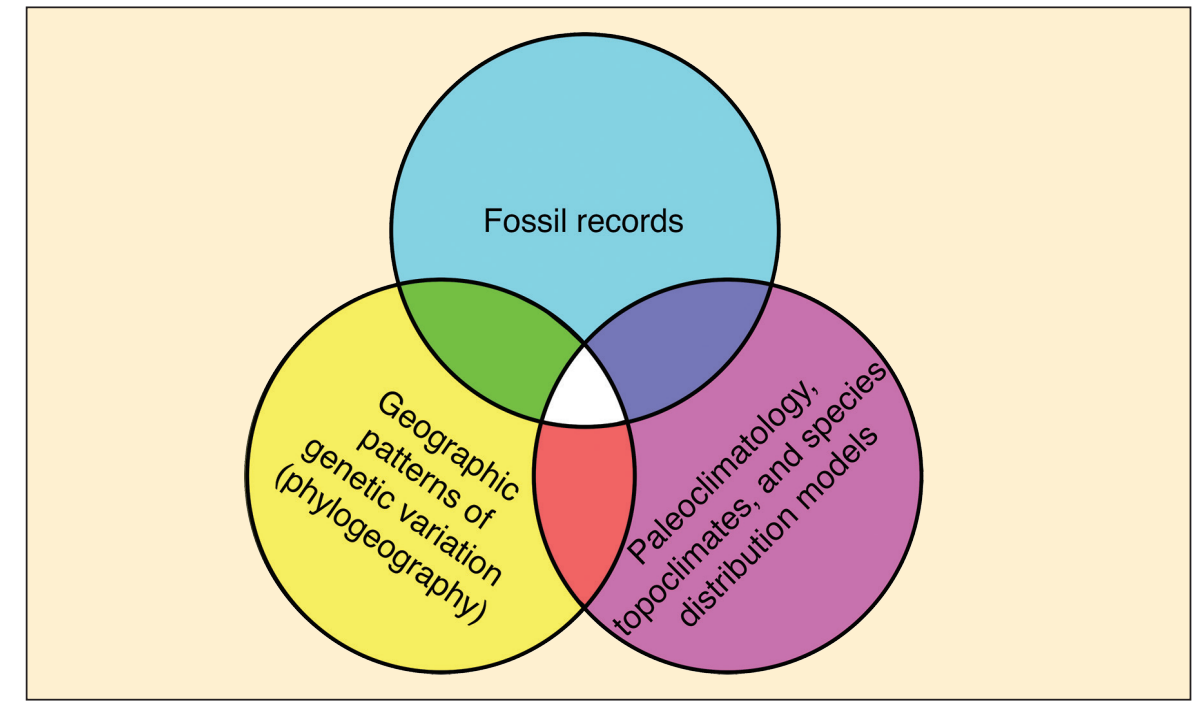

Figure 1: Three broad approaches to studying past species responses to climate change may be used jointly to exploit the complementary strengths of each approach to obtain the most complete reconstruction of historical refugia.

biodiversity research, and species distribution modeling. Three days of meetings were used to discuss advances within each discipline and opportunities for transdisciplinary research approaches.

There remains much untapped potential to study climate refugia by using a joint inference across multiple disciplines (Fig. 1). Joint analysis of data across disciplines enables a clearer and more robust picture of species history, as the strengths of the different disciplines are combined. For instance, the fossil record can be used to evaluate the performance of paleoclimate simulations and species distribution models (Varela et al. 2011). Second, hindcasted species distributions have been used to generate hypotheses that can be tested by phylogeographic studies (Richards et al. 2007), or simply to reveal past range dynamics that can explain the current distribution of genetic lineages (e.g. Hugall et al. 2002). The successful application of species distribution modeling to the study of refugia requires not only reliable paleoclimate estimates from coarse-resolution models, but also realistic downscaling of relevant variables over complex terrain in the present and the past (Dobrowski 2011). Phylogeography can notably help to detect small populations (cryptic refugia) and reveal the direction of past population expansions that went undetected in the fossil record, whereas the fossil record can constrain the timing of such expansions and detect past range contractions (i.e. local extinctions; Hu et al. 2009). Where sufficient data exist, a network of fossil sites may be interpreted with respect to the refugia inferred from genetics to constrain the spatial and temporal patterns of postglacial expansion, as has been done for Fagus sylvatica in Europe (Magri et al. 2006). Few studies to date have attempted joint inferences across all three approaches, and a quantitative framework that considers the strengths and weaknesses of each approach is needed for rigorous assessment of past species refugia and migration patterns. Such studies are likely to provide the most informed view of how individual species persisted through the Quaternary and, potentially, into the future.

\section{Selected references}

Full reference list online under:

http://www.pages-igbp.org/products/newsletters/ref2012_2.pdf

Dobrowski SZ (2011) Global Change Biology 17: 1022-1035

Hampe A, Jump AS (2011) Annual Review of Ecology, Evolution, and Systematics 42: 313-333

Hu FS, Hampe A, Petit RJ (2009) Frontiers in Ecology and the Environment 7:371-379

Keppel G et al. (2012) Global Ecology and Biogeography 21: 393-404 Magri D et al. (2006) New Phytologist 171: 199-221 